\title{
Effect of pressure on the characteristics of lifted flames
}

\author{
Seunghwan Bang ${ }^{1}$, Byeong Jun Lee ${ }^{1 *}$, Suk Ho Chung ${ }^{2}$ \\ ${ }^{1}$ School of Mechanical Engineering, Yeungnam University, Korea 38541 \\ ${ }^{2}$ King Abdullah University of Science and Technology (KAUST), Clean Combustion \\ Research Center (CCRC), Thuwal, Saudi Arabia 23955-6900
}

\section{Corresponding author:}

Byeong Jun Lee

School of Mechanical Engineering, Yeungnam University, Korea

Email: bjlee@ynu.ac.kr

\section{Colloquium: Laminar Flames}




\title{
Effect of pressure on the characteristics of lifted flame
}

\author{
Seunghwan Bang ${ }^{1}$, Byeong Jun Lee ${ }^{1 *}$, Suk Ho Chung ${ }^{2}$ \\ ${ }^{1}$ School of Mechanical Engineering, Yeungnam University, Korea 38541 \\ ${ }^{2}$ King Abdullah University of Science and Technology (KAUST), Clean Combustion \\ Research Center (CCRC), Thuwal, Saudi Arabia 23955-6900
}

\begin{abstract}
The effects of pressure on the characteristics of lifted flames in a coflow with propane fuel were investigated experimentally in a pressure chamber. Changing the pressure influenced the density, reaction kinetics, and flame propagation speed. The pressure range tested was $P=$ $0.5-5.5 \mathrm{~atm}$. As the fuel jet velocity increased, a nozzle-attached flame transitioned to a lifted flame before blowout occurred. Depending on pressure, the onset conditions of liftoff and blowout occurred in the laminar, transition, or turbulent regimes. When $P<1.6 \mathrm{~atm}$, the flame was lifted and had a tribrachial edge structure in the laminar regime, and the liftoff height $\left(H_{\mathrm{L}}\right)$ increased with increasing pressure. Both the liftoff and blowout velocities decreased with decreasing pressure, and they merged at $0.5 \mathrm{~atm}$. A correlation was derived in terms of the Schmidt number (Sc) and the Reynolds number (Re): $P^{n / 2}\left(H_{L} P^{n / 2}\right)^{(S c-1) /(2 S c-1)} \propto$ Re. The reattachment velocity in the laminar and transition regimes linearly decreased with pressure. The liftoff height had two local minimum points at a given Reynolds number in the transition regime, and then increased linearly in the turbulent regime.
\end{abstract}

Keywords: lifted flame, pressure, laminar flame, transition, turbulent flame

*Corresponding author: bjlee@ynu.ac.kr 


\section{Introduction}

Flame stabilization is an important safety issue in burner systems, and understanding the underlying physical mechanisms is essential. In turbulent lifted jet flames, several models have been proposed for the stabilization mechanisms, including the premixed flame model, the laminar flamelet model, the eddy dissipation model, and the large-scale mixing model, as reviewed by Pitts [1]. Also, secondary vortices at the flame base [2,3] may play an important role in flame anchoring. Of the models listed above, the premixed flame model [4], which states that a turbulent lifted flame is stabilized in the position where flow velocity and flame propagation velocity are balanced $[5,6]$, successfully explains various observations.

The laminar lifted flames theory is based on a tribrachial (triple) edge structure at the flame base consisting of both lean and rich premixed flame wings and a trailing diffusion flame coexisting at the flame edge $[7,8]$. The tribrachial structure dictates that (1) the edge is located along a stoichiometric contour due to the coexistence of three types of flames, and (2) the premixed flame wings have a certain propagation speed. Satisfactory correlations were obtained between the liftoff height and the jet velocity, based on a balance between the propagation speed of the edge flame and the local axial flow velocity along the stoichiometric contour of the laminar jet. Due to the nature of flow and concentration fields in jets, the Schmidt number of the fuel plays an important role in the existence of laminar lifted flames in free jets $[7,8]$.

Ambient pressure also directly effects density, transport properties, mass burning rate, and jet momentum and is, therefore, expected to have a significant influence on various combustion characteristics, such as flame propagation speed, flame thickness, and soot generation [9]. For example, the laminar burning velocity $\left(\mathrm{S}_{\mathrm{L}}\right)$ depends on the pressure $(P)$ according to the relation $S_{L} \propto P^{\frac{n-2}{2}}$ [9], where $n$ is the reaction order, typically 1.75 for propane [11], at which the laminar burning velocity decreases with pressure [10]. 
Considering that the balance between the flame propagation speed and the flow velocity influences lifted flame behaviors, liftoff height would be affected by variations in pressure.

Studies about the effect of pressure on lifted flames are very limited. The behavior of turbulent lifted jet flames in a pressure range of $50-100 \mathrm{kPa}$ was studied by Wang et al. [11], who focused on the blowout conditions. A global correlation for liftoff height was proposed as [12]

$$
P_{r}^{n-1} \frac{\rho S_{\mathrm{L}} H_{\mathrm{L}}}{\mu} \propto\left(\frac{u_{0}}{S_{\mathrm{L}}}\right) g(\bar{\rho})
$$

where $\rho$ is the fuel density, $H_{\mathrm{L}}$ is the liftoff height, $\mu$ is the fuel viscosity, $u_{0}$ is the fuel jet velocity, $g(\bar{\rho})=0.04+0.46 \bar{\rho}+0.5 \bar{\rho}^{2}$ is the density parameter in the reaction zone, $\bar{\rho}=$ $\rho_{\mathrm{e}} / \rho_{\infty}$, the reduced pressure $P_{\mathrm{r}}$ is defined as $P_{\mathrm{r}}=P / P_{s t}$, and the subscripts e and st indicate the fuel and standard atmospheric pressure, respectively. Since the reaction order $n$ is larger than 1 , the liftoff height is inversely proportional to the pressure. The effects of pressure on the characteristics of laminar lifted flames have not been reported yet. Although the liftoff height in the jet transition regime is known to decrease with jet velocity [13], the effect of pressure in this regime has not been clearly identified. The objective of the present study is to investigate the effects of pressure on liftoff height, lifted flame behavior, and blowout in the laminar, transition, and low-Reynolds-number turbulent regimes.

\section{Experiment}

The experimental apparatus consisted of a coflow burner, a pressure chamber, a pressure regulating system, a flow control system, and a measurement setup, as shown schematically in Fig. 1. The pressure chamber was made of stainless steel and had an inner diameter of $D=$ $200 \mathrm{~mm}$ and a height of $360 \mathrm{~mm}$. To visualize the lifted flames and to measure the liftoff height, two optical windows with a height of $200 \mathrm{~mm}$ and a width of $60 \mathrm{~mm}$ were installed. 
The coflow burner was placed inside the pressure chamber. A stainless steel fuel nozzle with an inner diameter of $D=0.22 \mathrm{~mm}$ was inserted at the center of the burner. Coflow air with a diameter of $92 \mathrm{~mm}$ was passed through wire meshes, stainless steel beads, and honeycomb to provide a near-uniform laminar flow of coaxial air. The chamber pressure was regulated with a pressure transducer, a solenoid valve, and a vacuum pump. To maintain stable pressure conditions inside the chamber, a micro needle valve was installed after the solenoid valve. The flow control system was composed of an air compressor and mass flow controllers. The liftoff height was measured using a cathetometer, and the flame images were taken on a digital camera.

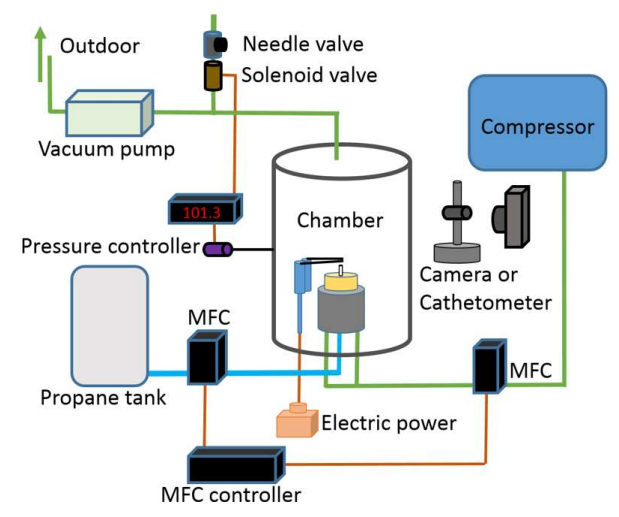

Fig. 1. Schematic of experimental setup.

House air and propane (99.9\% purity) were used as the coflow air and the fuel, respectively. The coflow air velocity was fixed at $0.06 \mathrm{~m} / \mathrm{s}$, which guaranteed a lean operation of the system for an air flow rate over 10 times the stoichiometric requirement at the maximum fuel flow rate. The ambient temperature was $24^{\circ} \mathrm{C}$. The solenoid valve and vacuum pump were connected to the pressure chamber. The other end of the solenoid valve was open to the air. The chamber pressure was adjusted to the desired value by controlling the solenoid valve; the operating pressure range was $P=0.5-5.5 \mathrm{~atm}$, covering both sub-atmospheric and pressurized conditions. A flame was initiated with an electrical spark ignitor and, after ignition, the ignitor were moved away so they would not disturb the flow fields. 
The liftoff height was monitored while increasing the fuel jet velocity at a specified chamber pressure. The laminar liftoff heights were measured by the cathetometer since they were very stable. In the transition and turbulent regimes, the liftoff height was determined from the average of 30 digital images. The lowest point that has higher intensity than the ambient signal along the centerline was defined as an instantaneous liftoff height.

\section{Results and Discussion}

\subsection{Flame shape}

Figure 2 shows typical flame images (captured on a digital camera) exhibiting different flame shapes and liftoff heights when the fuel jet velocity $u_{0}$ is varied under several pressures. Here, the Reynolds number is defined as $\operatorname{Re}=\left(u_{0} D / v\right)$, where $v$ is the kinematic viscosity of fuel. At $P=1.2 \mathrm{~atm}$ (a) and with low jet velocities, the flame is still attached to the nozzle and a sooting zone exhibiting a yellow luminous region near the tip of the diffusion flame can be observed. At a certain jet velocity, the flame lifts off, and the liftoff height increases with the jet velocity. The luminous yellow sooting zone disappears as the air and fuel mix in the upstream region of the lifted flame. At an excessive jet velocity, flame blowout occurs. When $P=1.2 \mathrm{~atm}$, the liftoff and blowout occur in the laminar regime. Although not clearly visible in the images, the lifted flame edge exhibits a tribrachial structure that can be identified with the naked eye. The structure has a bright and rich premixed flame inside, a small and lean premixed flame outside, and a faint blue diffusion flame, which coexist at the base of the flame. The liftoff height increases nonlinearly with the jet velocity, which will be discussed further below. The flame shows nearly flat-flame characteristics (close to $u_{0}=12.2 \mathrm{~m} / \mathrm{s}$ ) at blowout, similar to cases observed previously $[7,8]$.

After the initial liftoff of a nozzle-attached flame under $P=2.4 \mathrm{~atm}(\mathrm{~b})$, the liftoff height first decreases and then increases with the jet velocity. Although the flame is lifted off from the nozzle, a sooting zone still exists near the tip of the lifted flame due to both the small 
liftoff height (compared the case of $P=1.2 \mathrm{~atm}$ ), which causes insufficient mixing, and the effect of pressure on soot formation. The sooting zone diminishes with the jet velocity due to a decrease in the characteristic flow time. With increasing jet velocity, the flame base becomes wider, the flame fluctuates more, and blowout occurs near $u_{0}=14 \mathrm{~m} / \mathrm{s}$. The decreasing liftoff height with increasing jet velocity is a typical behavior of jet flames in the transition regime, due to a decrease in the jet breakup length, as observed previously [13]. This point will be further discussed later.

At $P=5$ atm (c), as the jet velocity increases, the liftoff height first increases $\left(u_{0}=7.1-8.4 \mathrm{~m} / \mathrm{s}\right)$, then decreases, and increases again when the jet becomes fully turbulent with the Reynolds numbers larger than 2300. Fluctuations near the flame base become strong just before blowout. The sooting region enlarges with the increased pressure.

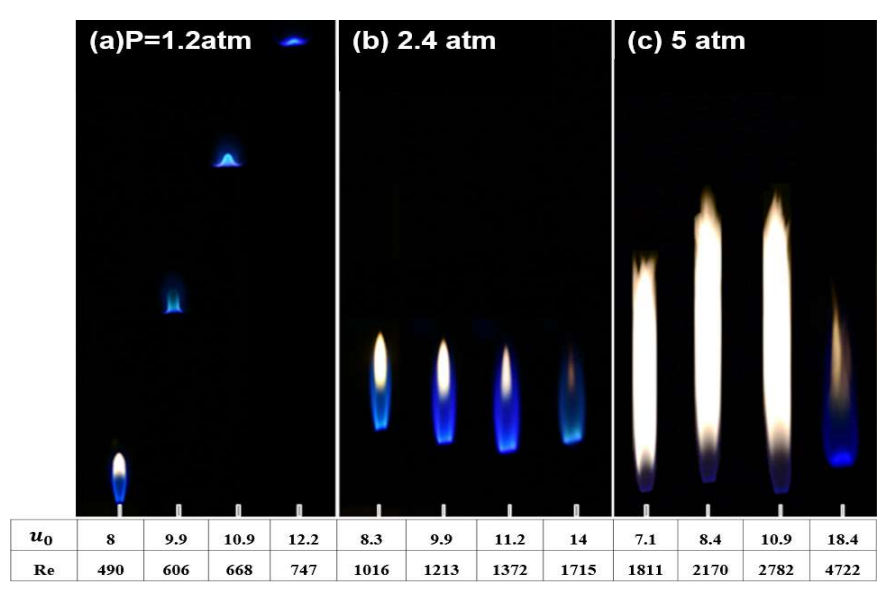

Fig. 2. Images of flames with varying jet velocities $\left(u_{0}[\mathrm{~m} / \mathrm{s}]\right)$ at several pressures.

\subsection{Liftoff height behavior}

Variations in liftoff height with increasing jet velocity under several different pressures, ranging from sub-atmospheric to pressurized conditions, are shown in Fig. 3 for lifted flames with a tribrachial structure in the laminar regime (a) and in the transition and turbulent regimes (b). The behavior of the liftoff height was consistent whether the burner was placed inside or outside of the pressure chamber. This can be attributed to the fact that the coflow 
diameter (over $400 D$ ) is sufficiently large compared with the fuel nozzle diameter $(D)$.

A lifted flame will move suddenly to the nozzle tip as the jet velocity decreases to the reattachment velocity [14]. Typically, the reattachment velocity is smaller than the liftoff velocity at a specified pressure. This can be attributed to the thermal and radical interactions between the flame and the nozzle in a nozzle-attached flame, which are absent in a lifted flame. The region between the liftoff and reattachment conditions is defined as the hysteresis region. The liftoff heights, including the hysteresis region, are plotted in Fig.3 until blowout. At a given pressure, the minimum and maximum jet velocities correspond to the reattachment and blowout conditions, respectively.

In the laminar regime (Fig. 3a), the liftoff height increases nonlinearly with the jet velocity for $P=0.5-1.6 \mathrm{~atm}$. Also, the liftoff height increases with the pressure at a specified jet velocity. Starting from the $P=1$ atm case, as the pressure increases, the range of jet velocities at which lifted flames exist shrinks, meaning that the difference between the reattachment and blowout velocities is reduced. Similarly, as the pressure decreases below 1 atm, the difference also decreases. This point will be further discussed later.

For $P \geq 1.8 \mathrm{~atm}(\mathrm{~b})$, the liftoff heights are smaller than $30 \mathrm{~mm}$, much smaller than those of laminar lifted flames. At $P=1.8 \mathrm{~atm}$, the liftoff height decreases with increasing fuel jet velocity until then a blowout occurs, similar to previous observations of nitrogen-diluted propane/air flames [13] in the transition regime. For $P \geq 3.6 \mathrm{~atm}$, the liftoff height increases, decreases, and then increases again with increasing jet velocity.

The relation between the laminar liftoff height and jet velocity was derived previously $[7,8]$ from similarity solutions for the axial velocity and fuel concentration. The following equation is derived from the balance between the propagation speed of the edge flame (assumed to be constant) and the local axial velocity along the stoichiometric contour: 

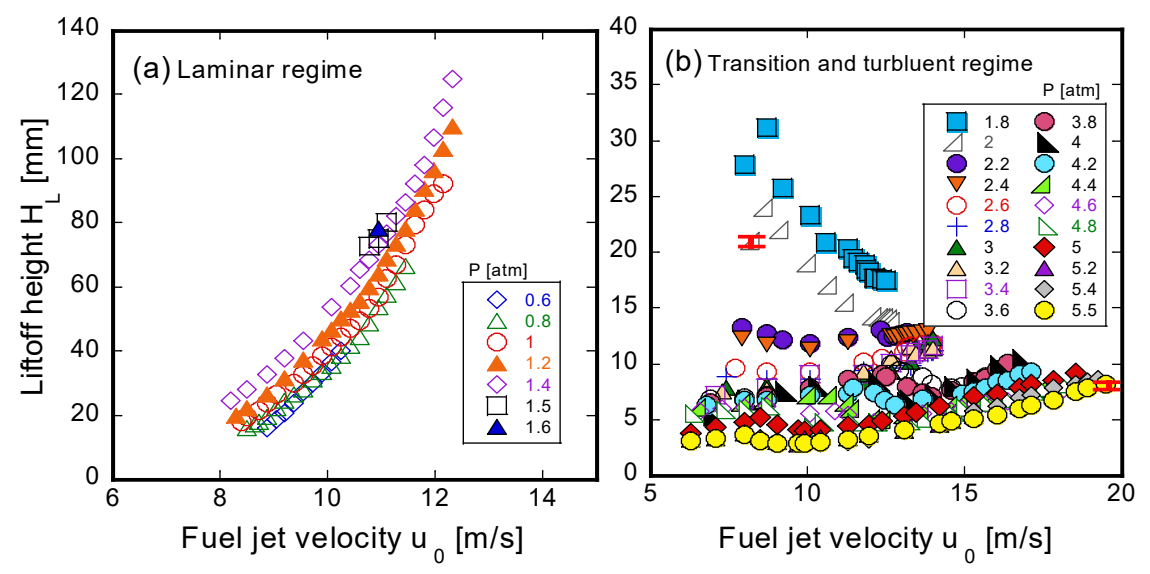

Fig. 3. Liftoff height variation with fuel jet velocity: (a) laminar regime for $P \leq 1.6 \mathrm{~atm}$ and (b) transition and turbulent regimes for $P \geq 1$.8atm (typical fluctuation range is marked in red bar).

$$
\left(\frac{H_{\mathrm{L}}}{D^{2}}\right)\left(\frac{v}{S_{\mathrm{e}}}\right)=\left\{\frac{3}{8(3+j)}\right\} \times\left\{\frac{12 Y_{\mathrm{F}}^{*}}{(3+j)(2 \mathrm{Sc}+1)}\right\}^{1 /(\mathrm{Sc}-1)} \times\left(\frac{u_{0}}{S_{\mathrm{e}}}\right)^{(2 \mathrm{Sc}-1) /(\mathrm{Sc}-1)}
$$

Here, $j$ is the constant geometric factor depending on flow configuration ( 0 for fully developed and 1 for uniform flow), $Y_{F}^{*}$ is the stoichiometric fuel mass fraction, Sc is the Schmidt number (defined as $v / D_{F}$ ), and $D_{F}$ is the fuel diffusivity. The edge-flame propagation speed $\left(\mathrm{S}_{\mathrm{e}}\right)$ is closely related to the stoichiometric laminar burning velocity $\left(S_{L}\right)$. Equation (2) implies that the pressure influences the liftoff height $\left(\mathrm{H}_{\mathrm{L}}\right)$ through the kinematic viscosity $v \propto P^{-1}$ and the laminar burning velocity $S_{L} \propto P^{(n-2) / 2}$.

The Schmidt number $(\mathrm{Sc})$ is generally insensitive to pressure. At a specified pressure, $v$ and $S_{L}$ can be treated as constants; therefore, Sc can be experimentally determined from Eq. (2) by curve fitting the liftoff height $\left(H_{\mathrm{L}}\right)$ to the exponent of the jet velocity $\left(u_{0}\right)$ [15]. The estimated Sc increases with pressure: 1.37 (0.8atm), 1.41 (1atm), 1.44 (1.2atm), and 1.46 (1.4atm). Since Sc only varies $4 \%$, Sc is assumed constant at 1.41 , its value under $1 \mathrm{~atm}$.

To further elucidate the effect of the pressure, Eq. (2) is rearranged as 


$$
P^{n / 2}\left(H_{\mathrm{L}} P^{n / 2}\right)^{(\mathrm{Sc}-1) /(2 \mathrm{Sc}-)} \propto \mathrm{Re} .
$$

Following Eq. (3), Fig. 4 shows the laminar liftoff height as a function of the Reynolds number, including the effect of pressure for $0.5 \leq P \leq 1.6 \mathrm{~atm}$. The reaction order $n=1.75$ was used [11]. The result shows an excellent linear correlation (the correlation coefficient $R^{2}$ $=0.998)$, regardless of the pressure variation, defined as

$$
P[\mathrm{~atm}]^{n / 2}\left(H_{\mathrm{L}}[\mathrm{mm}] P^{n / 2}\right)^{(\mathrm{Sc}-1) /(2 \mathrm{Sc}-1)}=0.085 \times \mathrm{Re}+9.32 .
$$

The result clearly indicates the effect of pressure on the laminar liftoff height in terms of the nondimensional Reynolds number, which represents the effect of fuel jet velocity in the laminar regime.

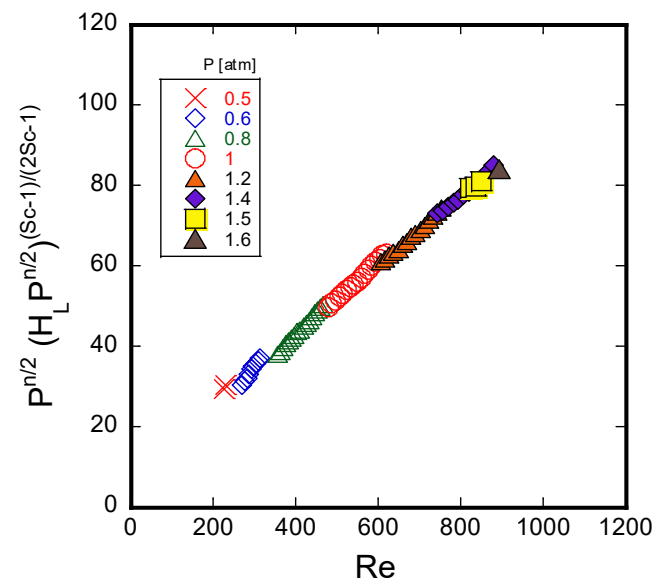

Fig. 4. Liftoff height $\left(H_{\mathrm{L}}[\mathrm{mm}]\right)$ correlation with the Reynolds number, including the effect of pressure $(\mathrm{P}[\mathrm{atm}])$ in the laminar regime.

In the turbulent regime, Kalghatgi [16] proposed an equation in explaining turbulent liftoff height characteristics. Recently, a global correlation for liftoff height was proposed [12] as 
was shown in Eq. (1).

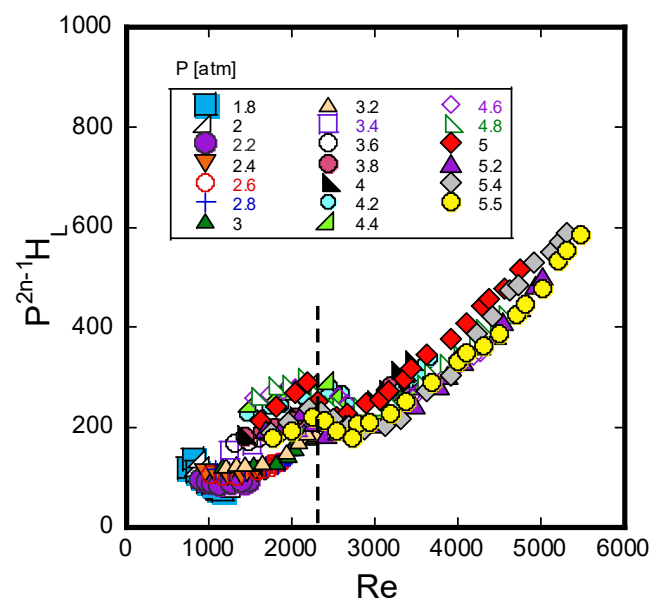

Fig. 5. Liftoff height $\left(H_{\mathrm{L}}[\mathrm{mm}]\right)$ correlation with Reynolds number in the transition and turbulent regimes (vertical dotted line corresponds to $\mathrm{Re}=2300$ ).

After the jet velocity $\left(u_{0}\right)$ is converted to a Reynolds number $(\mathrm{Re})$, and using the relations $\rho \propto P, v \propto P^{-1}$, and $S_{L} \propto P^{(n-2) / 2}$, Eq. (1) becomes

$$
P^{2 n-1} H_{\mathrm{L}} \propto \operatorname{Re}
$$

Figure 5 shows the correlation of the liftoff height in terms of the Reynolds number, including the effect of pressure in the transition and turbulent regimes with $n=1.75$. A reasonable correlation is exhibited. It is interesting that there are two local minimum points in the liftoff height behavior around $\mathrm{Re}=1200$ and 2600, and one local maximum point near $\mathrm{Re}$ $=2300$, which is the transition Reynolds number for fully developed pipe-flow conditions. This double-dip behavior has never been reported previously. This can be partially explained by the flow fluctuations in the jet stream from jet breakup (development of instability after ejecting from the nozzle) combined with the transition to turbulence inside the nozzle. To fully understand the double-dip behavior, a detailed investigation is needed in the future.

\subsection{Liftoff, blowout, and reattachment conditions}


Figure 6(a) shows the behaviors of the critical velocities. The liftoff and blowout velocities are determined by increasing the fuel jet velocity and the reattachment velocity by decreasing the jet velocity to create a transition from lifted to nozzle-attached flames.

The whole pressure range shown in Fig. 6(a) can be divided into five regions according to the flame behavior under liftoff (circle), blowout (triangle), and reattachment (square) conditions. Here, the blue symbols indicate the laminar regime, green indicates the transition regime, and red is the turbulent regime. Region I is where liftoff, reattachment, and blowout occur in the laminar regime. As the pressure decreases below $0.5 \mathrm{~atm}$, these three critical velocities merge. This implies that, as the pressure further decreases, laminar lifted flames do not exist; thus, $0.5 \mathrm{~atm}$ is the critical pressure for laminar lifted flames to exist. In region II, liftoff occurs in the laminar regime, while blowout occurs in the transition regime. Region III corresponds to liftoff and blowout mostly occurring in the transition regime. In region IV, the liftoff velocity decreases with pressure, and blowout occurs in the turbulent regime. In region $\mathrm{V}$, liftoff and blowout occur in the turbulent regime.

Although Fig. 6a is drawn following the experimental procedure, i.e., changing velocity while maintaining a specified pressure, it easier to identify clearly the laminar, transition, and turbulent regimes by plotting the results in terms of the Reynolds number, as shown in Fig. 6b. The turbulent blowout velocity depends linearly with pressure (can be fitted with $R^{2}=$ 0.999). Note that $\operatorname{Re}=u_{0} d / v \propto u_{0} P$ since $v \propto P^{-1}$. With this relationship, the turbulent blowout velocity can be a parabolic function of Re, which can be confirmed by a fitting. The difference between the liftoff and blowout conditions in the transition regime for $1000<\operatorname{Re}<$ 2600 is relatively small compared with other regimes, implying a narrow stable range in terms of jet velocity. 

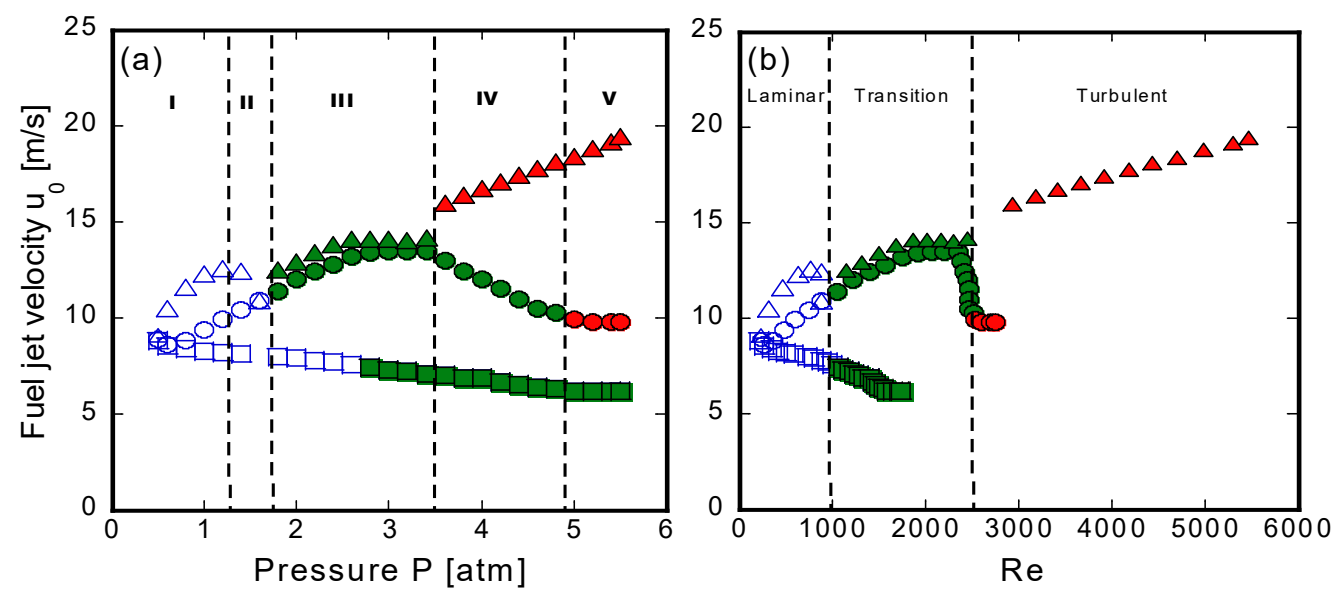

Fig. 6. Liftoff (circle), blowout (triangle), and reattachment (square) velocities in terms of (a) pressure and (b) Reynolds number (blue symbols for laminar regime, green for transition regime, and red for turbulent regime).

The liftoff velocity increases and then decreases with pressure, and the blowout velocity generally increases. The reattachment velocity $\left(u_{\mathrm{RA}}\right)$ has a unique characteristic: it decreases reasonably linearly with pressure (and also with $\mathrm{Re}$ ) and has the following correlation:

$$
u_{\mathrm{RA}}[\mathrm{m} / \mathrm{s}]=8.85-0.51 \times P[\mathrm{~atm}] .
$$

For $P<2.6 \mathrm{~atm}$, the liftoff velocity increases with pressure, whereas the reattachment velocity decreases. The liftoff velocity is nearly constant in the pressure range 2.8-3.2atm. Between 3.4 and 4.8atm, liftoff occurs near $\mathrm{Re}=2500$. In the turbulent regime with $P>5 \mathrm{~atm}$, the liftoff velocity is minimally affected by pressure.

The blowout velocities in each region have the following characteristics. In region I, blowout occurs in the laminar regime for $\operatorname{Re}<1000$, and the blowout velocity $\left(u_{\mathrm{BO}}\right)$ increases with pressure. This can be explained by the following relation, predicted by Lee and 
Chung [8], which defines the conditions satisfying the balance between edge propagation speed and axial velocity along the axis of a jet:

$$
\frac{3 \mathrm{Y}_{F}^{*}}{2 \mathrm{Sc}+1} \frac{u_{\mathrm{BO}}}{S_{e}}=1, \text { for } \mathrm{Sc}>1
$$

This condition implies a tribrachial point located along the centerline of the jet, and corresponds to a maximum jet velocity that simultaneously satisfies the stoichiometry and Se $=u_{\mathrm{L}}$ at the tribrachial point, where $u_{\mathrm{L}}$ is the local axial velocity. Using $S_{\mathrm{e}} \propto S_{\mathrm{L}}, S_{L} \propto$ $P^{(n-2) / 2}$, and the laminar burning velocity of propane under $1 \mathrm{~atm}(0.39 \mathrm{~m} / \mathrm{s})$ [17], Fig. 7 shows pressure effects on laminar blowout velocities and the relation of Eq. (7). Although there are some variations, the values are close to unity. Note that the free jet theory which we have used, assumes without having coflow air and without having buoyancy effect, such that the deviations could come from flow effect.

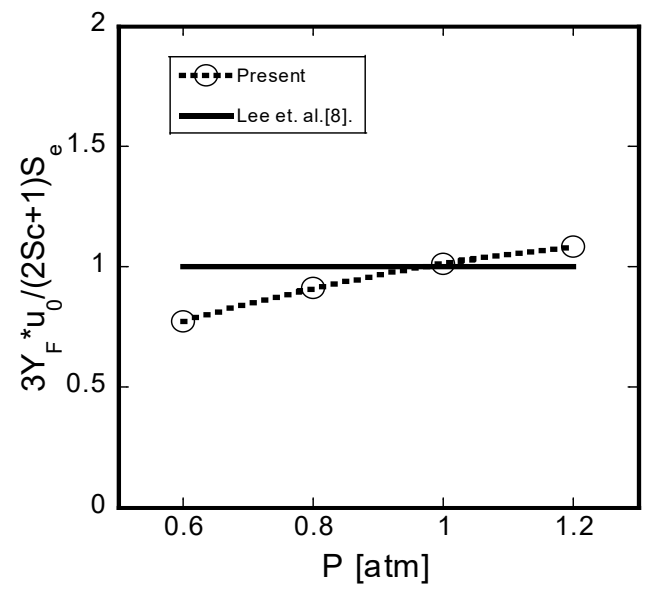

Fig. 7. Blowout conditions from region I in Fig. 6a.

In region I, the flame at blowout has a nearly flat shape, as shown in the last picture of Fig. 
2a. In region II, blowout occurs with Re smaller than 1000, but the blowout velocity decreases sharply with increasing pressure and Re, as shown in Fig. 6. This is because the blowout occurs having a non-flat flame shape with some fluctuations. To elucidate the reasons behind this non-flat flame blowout mode, the flow fields are visualized using the Schlieren method in Fig. 8 under several pressures. At $P=1.3 \mathrm{~atm}$, disturbances in the jet stream are not observed in the upstream region of the flame. In contrast, at 1.5 and 1.6atm, irregular disturbances exist in the upstream region, disturbing the flame and causing the flame to blowout in a non-flat flame mode. The occurrence of disturbances in the low Reynolds number was reported by Scholefield and Garside [18].

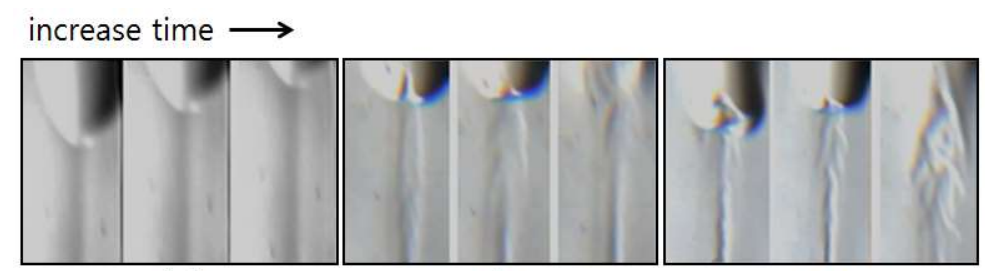

(a) (b)

(c)

Fig. 8. Schlieren images near the flame base during blowout at several pressures: (P[atm],

$$
\left.u_{0}[\mathrm{~m} / \mathrm{s}]\right)=(\mathrm{a})(1.3,12.8),(\mathrm{b})(1.5,11.4) \text {, and (c) }(1.6,10.9) \text {. }
$$

Region III is in the transition regime, where the blowout velocity increases with pressure and then levels off. The breakup length [13] can be defined as the distance from the nozzle tip to the point at which the jet-spreading angle changes appreciably, and can be seen in the Schlieren images. Figure 9 shows the breakup length and liftoff height at blowout conditions. For $P=1.8-3.4 \mathrm{~atm}$, these two values are close and decrease with pressure before leveling off at $P=3.4 \mathrm{~atm}$. This tendency is in accordance with the characteristics of propane-air flames diluted with nitrogen in the transition regime [13]. 


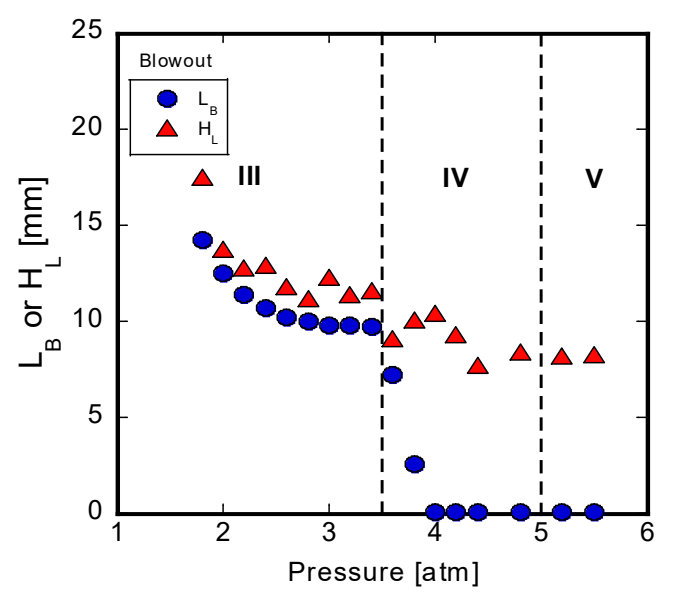

Fig. 9. Liftoff height $\left(H_{\mathrm{L}}\right)$ and breakup length $\left(L_{\mathrm{B}}\right)$ under blowout conditions in region III $(P$ $=1.8-3.4 \mathrm{~atm}), \mathrm{IV}(3.4-5 \mathrm{~atm})$, and V (> 5atm).

In regions IV and $\mathrm{V}$, the blowout occurs in the turbulent regime when $\operatorname{Re}>2600$, as manifested by the near-zero breakup length. The liftoff height at blowout remains reasonably constant independent of pressure.

The blowout velocity $\left(u_{\mathrm{BO}}\right)$ increases nearly linearly with pressure in the turbulent regime, as shown in Fig. 6. This is similar to a trend reported previously [11], where the blowout velocity of turbulent lifted flames was measured at sub-atmospheric conditions. There, the relationship between the pressure and blowout velocity [11] was deduced as

$$
u_{B O} \sim \frac{P_{r}^{n-1} d S_{L}^{2}[1 \mathrm{~atm}]}{\alpha[1 \mathrm{~atm}]\left(\rho_{e} / \rho_{0}\right)^{1 / 2} \mathrm{Da}}
$$

where $\alpha$ is the thermal diffusivity, $\rho_{0}$ is the ambient density, $\rho_{e}$ is the fuel density at the nozzle exit, and Da is the Damköhler number defined as the ratio of characteristic flow to reaction times, which was found to be 5.6 at blowout [11]. The effect of pressure on all the variables is defined by $P_{r}^{n-1}$, where $P_{r}=P / P_{s t}$ and $P_{s t}=1 \mathrm{~atm}$. Figure 10 shows both the 
measured blowout velocity from the current study and that calculated using Eq. (8) [11]; the $S_{\mathrm{L}}$ values were adopted from another study [17]. A reasonable agreement between the present measured and calculated using Eq. (8) [11] blowout velocities is observed, and the difference could be partially attributed to the pressure range, i.e., 0.5-1atm [11] and 3.6-5.5atm (the present study).

In the turbulent regime (Fig. 5), the liftoff height with Re has the best fit with $R^{2}=0.94$ with $\mathrm{n}=1.79$ for $4.0 \leq P \leq 5.5 \mathrm{~atm}$, which is in good agreement with the proposed $\mathrm{n}=1.75$. In the transition and turbulent regimes, the best fit is with $n=1.69$ which is also close to the propose $n=1.75$.

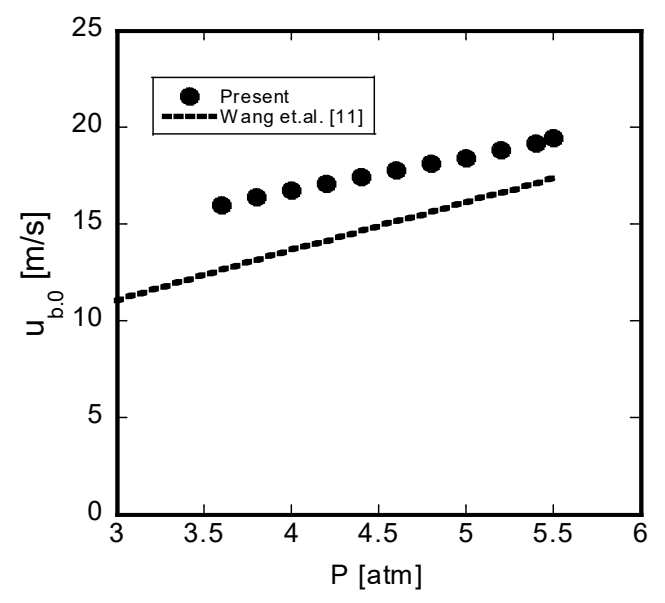

Fig. 10. Effect of pressure on blowout velocities in regions IV and V.

\section{Conclusions}

The flames with a nozzle size of $0.22 \mathrm{~mm}$ demonstrate all the observed flame characteristics, spanning from laminar liftoff to turbulent blowout, as pressure and fuel velocity varied. The conclusions obtained from this study are as follows:

(1) The effect of pressure on the laminar liftoff height can be explained by the equation for 
laminar tribrachial lifted flame expressed as

$$
P^{n / 2}\left(H_{\mathrm{L}} P^{n / 2}\right)^{\frac{S c-1}{2 S c-1}} \propto \operatorname{Re}
$$

(2) In the transition and turbulent regimes, the liftoff height has two local minimum points with the Reynolds number.

(3) The liftoff height in the transition and turbulent regimes has the following correlation

$$
P^{2 n-1} H_{\mathrm{L}} \propto \operatorname{Re}
$$

(4) The reattachment velocity decreases linearly with pressure in the laminar, transition, and turbulent regimes.

(5) With increasing Re, the liftoff velocity increases until the transition region, then sharply decreases near $\mathrm{Re}=2500$. After that, it is minimally affected by pressure.

(6) Disturbances in the jet stream when $\operatorname{Re}<1000$ for $P=1.5-1.6$ atm cause non-flat flames at blowout and the blowout velocity decreases with increasing $P$ or Re.

(7) The turbulent blowout velocity increases reasonably linearly with pressure. 


\section{References}

(1) W.M. Pitts, Proc. Combust. Inst 22 (1988), 809-816

(2) D. Demare, F. Baillot, Phys. Fluids 13 (2001), 2662-2670

(3) K.M. Lyons, K.A. Watson, J.M. Donbar, Combust. Sci. and Tech. 179 (2007), 1029-1037

(4) L. Vanquickenborne, T. Van, Combust. Flame 10 (1966) 59-69

(5) K.M. Lyons, K.A. Watson, J.M. Donbar, Combust. Sci. and Tech. 179 (2007), 1029-1037

(6) C. Iyogun, M. Birouk, Combust. Sci. and Tech. 180 (12) (2008), 2186-2209

(7) S.H. Chung, B.J Lee, Combust. Flame 86 (1991) $62-72$.

(8) B.J. Lee, S.H. Chung, Combust. Flame 109 (1997) 163-172

(9) S.R. Truns, An Introduction to Combustion, McGraw-Hill, p. 267-277, 2001

(10)E.S. Cho, T.K. Oh, S.H. Chung, Combust. Sci. Technol. 178 (2006) 1559-1584.

(11)Q. Wang, L. Hu, S.H. Chung, Combust. Flame 176 (2017) 358-360.

(12)Q. Wang, L. Hu, M. Zhang, F. Tang, X. Zhang, S. Lu, Combust. Flame 161 (2014) 1125-1130.

(13)B.J. Lee, J.S. Kim, S.H. Chung, Proc. Combust. Inst 25 (1994) 1175-1181.

(14) J. Lee, S.H. Chung, Combust. Flame 127 (2001) 2194-2204.

(15)B.J. Lee, and S.H. Chung, Fuel 85 (2006) 68-74.

(16) G.T. Kalghatgi, Combust. Sci. and Tech. 41 (1-2) (1984) 17-29

(17)X. Shi, J.Y. Chen, Y. Chen, Combust. Flame 176 (2017) 38-47

(18)D.A. Scholefield, J.E. Garside, Proc. Combust. Inst. 3 (1954) 102-110. 


\section{List of Figure Captions}

Fig. 1. Schematic of experimental setup.

Fig. 2. Images of flames with varying jet velocities $\left(u_{0}\right)$ at several pressures.

Fig. 3. Liftoff height variation with fuel jet velocity: (a) laminar regime for $P \leq 1.6 \mathrm{~atm}$ and (b) transition and turbulent regimes for $P \geq 1.8 \mathrm{~atm}$.

Fig. 4. Liftoff height $\left(H_{\mathrm{L}}: \mathrm{mm}\right)$ correlation with the Reynolds number, including the effect of pressure in the laminar regime.

Fig. 5. Liftoff height $\left(H_{\mathrm{L}}: \mathrm{mm}\right)$ correlation with Reynolds number in the transition and turbulent regimes (vertical dotted line corresponds to $\mathrm{Re}=2300$ ).

Fig. 6. Liftoff (circle), blowout (triangle), and reattaching (square) velocities in terms of (a) pressure and (b) Reynolds number (blue symbols for laminar regime, green for transition regime, and red for turbulent regime).

Fig. 7. Blowout conditions from region I in Fig. 6(a).

Fig. 8. Schlieren photographs near the flame base during blowout at several pressures: $\left(\mathrm{P}[\mathrm{atm}], u_{0}[\mathrm{~m} / \mathrm{s}]\right)=(\mathrm{a})(1.3,12.8)$, (b) $(1.5,11.4)$, and (c) $(1.6,10.9)$.

Fig. 9. Liftoff height $\left(H_{\mathrm{L}}\right)$ and breakup length $\left(\mathrm{L}_{\mathrm{B}}\right)$ under blowout conditions in region III $(P$ $=1.8-3.4 \mathrm{~atm})$, IV (3.4-5atm), and V (> 5atm).

Fig. 10. Effect of pressure on blowout velocities in regions IV and V. 